Article

\title{
Development of In-Situ Poled Nanofiber Based Flexible Piezoelectric Nanogenerators for Self-Powered Motion Monitoring
}

\author{
Minjung Kim ${ }^{1}$, Vignesh Krishnamoorthi Kaliannagounder ${ }^{1}$ (D) Afeesh Rajan Unnithan ${ }^{1,2}$, \\ Chan Hee Park $1,3, *$, Cheol Sang Kim ${ }^{1,3, *}$ and Arathyram Ramachandra Kurup Sasikala 1,4,*(D) \\ 1 Department of Bionanosystem Engineering, Graduate School, Jeonbuk National \\ University, Jeonju 561-756, Korea; alswjd2821@gmail.com (M.K.); vikkikrish8@gmail.com (V.K.K.); \\ afeesh@jbnu.ac.kr (A.R.U.) \\ 2 Healthcare Technology Institute, Institute of Translational Medicine, School of Chemical Engineering, \\ University of Birmingham, Edgbaston, Birmingham B15 2TT, UK \\ 3 Mechanical Design Engineering, Jeonbuk National University, Jeonju 561-756, Korea \\ 4 College of Medical and Dental Sciences, Sir Robert Aitken Institute for Medical Research, \\ University of Birmingham, Edgbaston, Birmingham B15 2TT, UK \\ * Correspondence: biochan@jbnu.ac.kr (C.H.P.); chskim@jbnu.ac.kr (C.S.K.); arathy@jbnu.ac.kr (A.R.K.S.)
}

Received: 26 March 2020; Accepted: 15 May 2020; Published: 18 May 2020

\begin{abstract}
Energy harvesting technologies have found significant importance over the past decades due to the increasing demand of energy and self-powered design of electronic and implantable devices. Herein, we demonstrate the design and application of in situ poled highly flexible piezoelectric poly vinylidene fluoride (PVDF) graphene oxide (GO) hybrid nanofibers in aligned mode for multifaceted applications from locomotion sensors to self-powered motion monitoring. Here we exploited the simplest and most versatile method, called electrospinning, to fabricate the in situ poled nanofibers by transforming non-polar $\alpha$-phase of PVDF to polar $\beta$ - phase structures for enhanced piezoelectricity under high bias voltage. The flexible piezoelectric device fabricated using the aligned mode generates an improved output voltage of $2.1 \mathrm{~V}$ at a uniform force of $12 \mathrm{~N}$. The effective piezoelectric transduction exhibited by the proposed system was tested for its multiple efficacies as a locomotion detector, bio-e-skin, smart chairs and so on.
\end{abstract}

Keywords: Poly vinylidene fluoride; Graphene oxide; Electrospinning; Polar $\beta$ - phase; Piezoelectricity; Nanogenerator

\section{Introduction}

Energy harvesting technologies have found significant importance over the past decades due to the increasing demand of energy and self-powered design of electronic and implantable devices [1-3]. The concept of self-powered smart electronics such as wearable devices for personal health care [4], biomonitoring [5] and environmental monitoring [6] necessitates unique, cost effective and sustainable energy storage systems [7]. The rapid progress in the field of nanotechnology has made it possible to effectively convert the mechanical energy into electrical energy by means of nanogenerators [7]. In 2006, Wang and co-workers introduced the piezoelectric nanogenerator (PENG) from $\mathrm{ZnO}$ nanowires to harvest electrical energy from mechanical stretching [8]. It opened a new room for researchers in PENG technologies to come up with quality research outcomes for power generation through biomechanical energy harvesting for practical applications [9-11].

PENGs exploit the material's property called piezoelectricity. Piezoelectric materials convert mechanical energy into electrical energy due to their unique crystallography. A non-central 
symmetric structure is an essential criterion for a material to be piezo electric to produce an electric dipole under stress [12]. Numerous remarkable organic and inorganic materials including lead magnesium niobate-lead titanate (PMN-PT) [13,14] lead zirconate titanate (PZT) $[15,16]$, barium titanate (BTO) $[10,17]$ and poly vinylidene fluoride (PVDF) [18] have been extensively studied as ferroelectric nanogenerators (FENGs). Though the inorganic materials possess higher piezoelectric characteristics, their brittle/rigid nature has posed concern about their durability [19]. On the other hand, ferroelectric polymers show enhanced flexibility with lower piezoelectricity [20]. They can sustain larger strains compared to their inorganic counterparts [21]. Therefore, various nanocomposites of inorganic piezoelectric materials in organic polymers have been implemented to impart flexibility and robustness along with improved performance [22,23].

Recently, polyvinylidene fluoride (PVDF) has gained ample attention in self-powered piezoelectric devices due to its incredible electroactive properties such as piezoelectricity, ferroelectricity and pyroelectricity with high chemical stability [24]. PVDF is an inexpensive, highly nonreactive and a flexible polymer [25] with four crystalline phases, namely $\alpha, \beta, \gamma$ and $\delta$, based on the chain formation [26-28]. Among these phases, $\alpha$ is non-polar and does not show any piezoelectricity. In order to enhance the piezoelectricity, the $\beta$ and $\gamma$ phases can be generated by nanoparticle doping [29], 2D metal dichalcogenides [7], electrospinning process [30] etc. In this regard, graphene oxide (GO) has shown extensive applicability due to its superior property to induce the nucleation of $\beta$ and $\gamma$ phases by the electrostatic interaction and/or hydrogen bonding between the oxygen functionalities of GO and PVDF as well as its superior mechanical, thermal and electrical properties [31].

Herein, we demonstrate the efficacy of an in situ poled highly flexible piezoelectric PVDF GO hybrid nanofibers in different alignment modes for multifaceted applications from locomotion sensor to signal monitoring. Here we exploited the simplest and most versatile method, called electrospinning, to fabricate the in situ poled nanofibers by transforming non-polar $\alpha$-phase of PVDF to polar $\beta$-phase structures for piezo-electricity under high bias voltage [32-34]. The flexible piezoelectric device fabricated using the aligned mode generates a highly enhanced output voltage of $2.1 \mathrm{~V}$ at a mechanical vibration frequency of $8 \mathrm{~Hz}$. The effective piezoelectric transduction exhibited by the proposed system enables its multiple efficacies as a locomotion detector, bio-e-skin, smart chairs and so on.

\section{Experimental Section}

\subsection{Materials}

$\mathrm{N}, \mathrm{N}$-dimethylformamide (DMF), acetone, PVDF (polyvinylidene fluoride), and polydimethylsiloxane (PDMS) were obtained from Sigma Aldrich Korea, and graphene oxide (GO) was purchased from Standard Graphene, Korea.

\subsection{Piezoelectric Nanofiber Fabrication}

The piezoelectric nanofibers were fabricated using the custom-made electrospinning setup and technique that we reported previously [35,36]. In this study, we prepared 17\% PVDF solution in DMF and acetone at a 2:1 ratio. The prepared PVDF solution was fed to the plastic syringe tube, which is connected to a metal capillary nozzle with a needle diameter of $0.21 \mathrm{~mm}$ and which is coupled to a $1 \mathrm{D}$ robot system that moves laterally and is controlled by the Lab View 9.0 program (National Instruments). The solution feed rate was maintained at $1 \mathrm{ml} / \mathrm{hr}$ with a fixed voltage of $20 \mathrm{KV}$ and the tip to collector distance was kept at $10 \mathrm{~cm}$ throughout the experiment. For creating aligned PVDF nanofibers, we used a custom made highspeed rotating collector and for making random fibres a normal rotating drum collector is used. Different concentrations (2,5 and $7 \mathrm{wt} . \%)$ of GO were added to the PVDF solution after sonication to prepare 2, 5 and $7 \mathrm{wt} . \%$ of GO-loaded PVDF solution for electrospinning. GO-PVDF electrospinning conditions were maintained same as described above throughout the experiment for both random and aligned nanofiber production. 


\subsection{Characterisation}

The morphology of the samples was observed by field emission scanning electron microscopy (FESEM; Zeiss Supra 40VP). X-ray powder diffraction (XRD) was carried out on a Rigaku X-ray diffractometer $(\mathrm{Cu} \mathrm{K} \alpha, \mathrm{k}=1.54059 \AA)$ over Bragg angles ranging from $10^{\circ}$ to $45^{\circ}$ to characterize the crystallinity and $\beta$ phase formation of the samples. The relative proportion of the $\beta$ phase formation was assessed from XRD by calculating the ratio of the peak intensity for $\beta\left(\mathrm{I}_{\beta}\right)$ and sum of peak intensities of $\alpha\left(\mathrm{I}_{\alpha}\right)$ and $\beta$, respectively, as given below [37].

$$
\frac{I_{\beta}}{I_{\alpha}+I_{\beta}}=\frac{I_{200} / I_{110}}{\left(I_{100}+I_{020}+I_{110}+I_{021}\right) \alpha+\left(I_{200} / I_{110}\right) \beta}
$$

where $\mathrm{I}_{100}, \mathrm{I}_{020}, \mathrm{I}_{100}, \mathrm{I}_{021}$ and $\mathrm{I}_{200} / \mathrm{I}_{110}$ correspond to the peak intensities of $(100),(020),(100),(021)$, and (200/110) planes, respectively.

In order to confirm the chemical structures and bonding configuration of the PVDF and nanocomposite fibers, FT-IR spectra (Paragon 1000 Spectrometer, Perkin Elmer) were obtained. The relative proportion of $\beta$ phase in the samples were also calculated from the FTIR as per the equation [37]:

$$
\frac{I_{\beta}}{I_{\beta}+I_{\alpha}}=\frac{I_{513}+I_{839}+I_{1272}}{I_{491}+I_{530}+I_{615}+I_{764}+I_{796}+I_{978}+I_{513}+I_{839}+I_{1272}}
$$

where $\mathrm{I}_{513}, \mathrm{I}_{839}, \mathrm{I}_{1272}, \mathrm{I}_{491}, \mathrm{I}_{530}, \mathrm{I}_{615}, \mathrm{I}_{764}, \mathrm{I}_{796}, \mathrm{I}_{978}, \mathrm{I}_{513}, \mathrm{I}_{839}$, and $\mathrm{I}_{1272}$ designate the peak intensity of adsorption bands at $513,839,1272,491,530,615,764,796,978,513,839$, and $1272 \mathrm{~cm}^{-1}$, respectively.

The differential scanning calorimetry (SDT Q600 V20.9 Build 20) thermograms of the PVDF nanocomposite were obtained to estimate the crystallization behaviour. The degree of crystallinity $\left(X_{\mathrm{C}}\right)$ was calculated by the following equation

$$
X_{C}=\frac{\Delta H_{m}}{\Delta H_{100 \%}} * 100
$$

where $\Delta \mathrm{H}_{\mathrm{m}}$ and $\Delta \mathrm{H}_{100 \%}$ refer to the heat of fusion of the sample and $100 \%$ crystalline.

Micro-Raman spectroscopy (Nanofinder 30) with an argon ion laser at an excitation wavelength of $532 \mathrm{~nm}$ was used to confirm the different structural phases of the GO in the samples.

\section{Piezoelectric Nanogenerator Device Fabrication and Piezoelectricity Measurements}

The PVDF NF-based ferroelectric nanogenerator (FENG) devices were fabricated according to the already established method. The NG was fabricated by taking the required dimension $(2 \times 2 \mathrm{~cm})$ of each NF sample and the copper electrode was sputtered on the top and bottom surface of the NF film. Two copper wires $(0.1 \mathrm{~mm})$ were attached with copper electrodes with the help of conducting silver paste and finally polydimethylsiloxane (PDMS) was used as a packaging layer for the NG to protect the device from external conditions.

The piezoelectric property of the samples was analysed by means of an experimental setup consisting of a frequency generator and an output measurement system. The sample was placed on the sample holder and the desired frequencies of vibration according to different mechanical loads were selected. As a result, the vibrating nob applies the desired frequency of vibration on top of the sample to induce electrical response. The output voltage generated by the sample was measured by a digital oscilloscope (Yokogawa, DL9040) to evaluate the piezoelectricity. Furthermore, the piezoelectric coefficient d33 for PVDF R, PVDF GO R, and PVDF GO A was calculated by using the equation [38].

$$
\mathrm{d}_{33}=\left(\mathrm{C}^{*} \mathrm{~V}\right) / \mathrm{F}
$$

where $\mathrm{F}$ is the applied force and $\mathrm{V}$ is the generated piezoelectric potential over a capacitor $(\mathrm{C}=70 \mathrm{pF})$ in parallel with the FENG device. 
In order to check the efficacy of the PEGAN FENG as piezoelectric sensors, the as prepared FENG device was attached to a finger, on the sandal and on the handle of a chair. The stress induced piezoelectricity was analysed as described above using the digital oscilloscope.

\section{Results and Discussion}

The schematic diagram shows the fabrication of piezoelectric nanofiber (Figure 1A). A facile and modest electrospinning technique was used to fabricate the electrospun piezoelectric PVDF mats in aligned and random orientation. It has been already reported that the nanofibers (NFs) alignment also play crucial role in determining the crystalline structure and thereby piezoelectric properties of the samples [39]. In order to enhance the piezoelectric $\beta$ phase nucleation, GO was selected as a filler due to its active interaction with the PVDF matrix as well as its higher dielectric constant. Different concentrations (2, 5 and $7 \mathrm{wt} . \%$ ) of GO were added with PVDF and electrospun to obtain the random (PGRNF) as well as aligned (PGANF) composite nanofibers. The optimization of the GO concentration has been done by analysing the surface morphologies of the composite nanofibers along with the pristine PVDF NF using the FESEM studies. The FESEM results reveals that the $5 \mathrm{wt} . \%$ GO encapsulated PVDF nanofibers showed better fibre morphology (Figure 1D,E) compared to the rest of the samples (Figure S1A-C of the supporting information (SI)). Moreover, the composite nanofiber possesses even rough morphology compared to the quite smooth surface of the pure PVDF nanofibers (Figure 1B,C), confirming the uniform encapsulation of GO throughout the nanofiber without any agglomeration. The pristine and composite nanofibers fabricated in aligned orientation significantly exhibits a highly ordered arrangement compared to their random counterparts, revealing the successful orientation of the NF in an aligned fashion, since our first priority was to fabricate the piezoelectric nanofibers with better morphology for higher piezoelectric performance. In case of nanofiber-based FENGs, the morphology plays a crucial role in determining their performance and hence the beaded nanofibers or distorted fiber morphologies will hinder the material performance [40,41]. Considering the results obtained, we selected $5 \mathrm{wt} . \% \mathrm{GO}$ encapsulated PVDF nanofibers as the final material for FENG fabrication.

The structural properties and crystallization behaviour of the NFs were confirmed by XRD and FTIR. The XRD pattern (Figure 2A) clearly shows that the PVDF powder possess the characteristic peaks of the non-polar $\alpha$ phase at $2 \theta=17.6,18.3,19.9$ and 26.5 corresponding to the reflection planes (100), (020), (110) and (021) planes [31]. However, a more intense single peak is seen at $2 \theta=20.4 \sim 20.6$, relating to the $(200) /(110)$ plane for the electrospun nanofiber samples, revealing the polarized $\beta$ phase formation after electrospinning. It is also observed that the peaks assigned to the $\beta$ phase are significantly stronger and sharper in the PGANF compared to the randomly oriented NFs. In order to confirm the $\beta$ phase enhancement due to the nanofiber alignment and GO addition, the relative proportion of the $\beta$ phase formation is assessed as per Equation (1) provided in the experimental section, and the relative $\beta$ phase formation is found to be highest in the PGANF ( 67\%) and lowest in the pristine PVDF NF (PNF) ( 55.7\%). PGRNF ( 59.4\%) exhibits an intermediate value for the relative $\beta$ phase formation.

Figure 2B represents the FTIR spectra of PVDF powered, pristine PVDF NF and the GO composite nanofibers in different alignments. The peculiar peaks associated with GO such as the bands at 1038, 1157 and $1356 \mathrm{~cm}^{-1}$ for the C-O stretching, C-O-C stretching, and the C-O-H deformation, respectively, are visible in both the composite nanofibers, clearly confirming the presence of GO in the NF system (Figure S2 of SI) [37]. The characteristic peaks for the $\alpha$ phase in PVDF at 491, 515, 530, 615, 746, 796, 839, 978 and $1272 \mathrm{~cm}^{-1}$ are clearly visible in the PVDF powder sample whereas the absorption bands for $\beta$ phase at 509, 839 and $1273 \mathrm{~cm}^{-1}$ started appearing for the electrospun sample (Figure 2B), showing the in situ polarization [37]. It is also seen that the addition of GO enhanced the $\beta$ phase nucleation due to the appearance of significantly strong $\beta$ phase peaks along with the indistinctive peaks of $\alpha$ phase. Thus, to understand the extent of polarization, the relative proportion of $\beta$ phase using the FTIR spectra was calculated according to Equation (2) and the relative proportion of $\beta$ phase was found to be increased as given: PVDF powder $(0.23)<$ Pristine PNF $(0.58)<$ PGRNF $(0.61)<$ 
PGANF (0.69). The obtained values for the relative proportion of $\beta$ phase obtained from both XRD and FTIR (Figure S3 of SI) reveals that the proportion of $\beta$ phase increased after the addition of GO into the PVDF. Moreover, the enhanced formation of $\beta$ phase over $\alpha$ phase was also strongly associated with the nanofiber alignment [39]. Thus, we strongly hypothesise that the PGANF will be an ideal candidate for piezoelectric sensors application.

Before going into the piezoelectric sensor studies, we further analysed the effect of electrospinning on GO in combination polymeric chains of PVDF has been done using the Raman spectroscopy technique. The typical GO peaks around 1324 (D band) and $1592 \mathrm{~cm}^{-1}$ (G band) [42] are clearly found in the Raman spectra of PGANF (Figure 2C), further confirming the presence of GO in the NF matrix. To further quantify the crystallinity, the differential scanning calorimetry studies were performed. The heating curve of the PGANF (Figure 2D) shows a peak melting temperature $\mathrm{T}_{\mathrm{m}}$ of $162.42{ }^{\circ} \mathrm{C}$. The crystallinity $\left(\chi_{c}\right)$ of the sample was calculated according to Equation (3) and the crystallinity of PGANF was found to be $56.27 \%$. Thus, according to the proportion of $\beta$ phase obtained from XRD, FTIR and a DSC thermograph, we concluded that the PGANF can be used as a good material for biomechanical piezoelectric sensors.

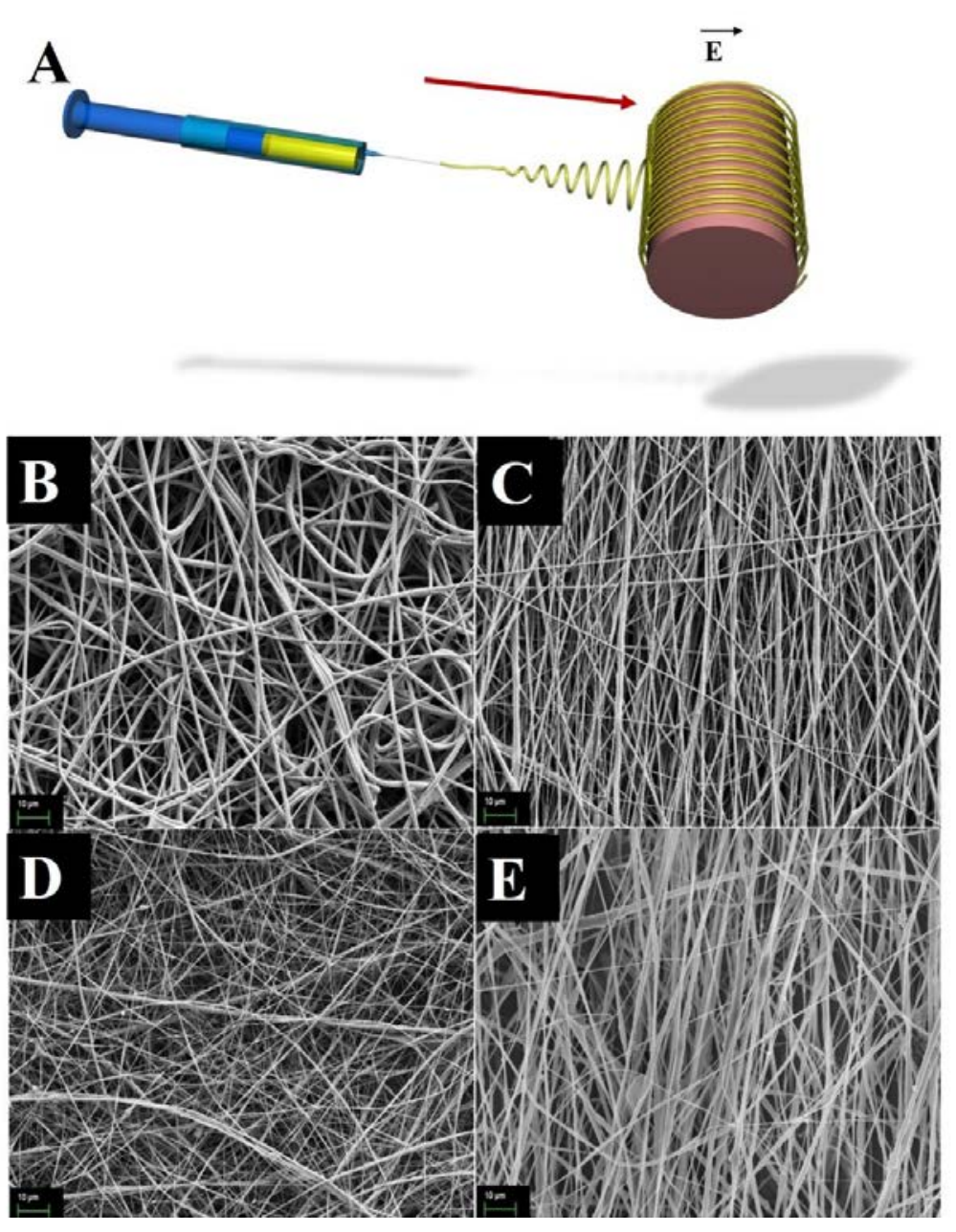

Figure 1. (A) Schematic illustration showing the electrospinning setup, field emission scanning electron microscopy (FESEM) image showing the (B) pristine poly vinylidene fluoride (PVDF) random nanofiber (NF) (PNF), (C) pristine PVDF aligned NF, (D) PVDF-graphene oxide (GO) random NF (PGRNF), (E) PVDF-GO aligned NF (PGANF). 
A
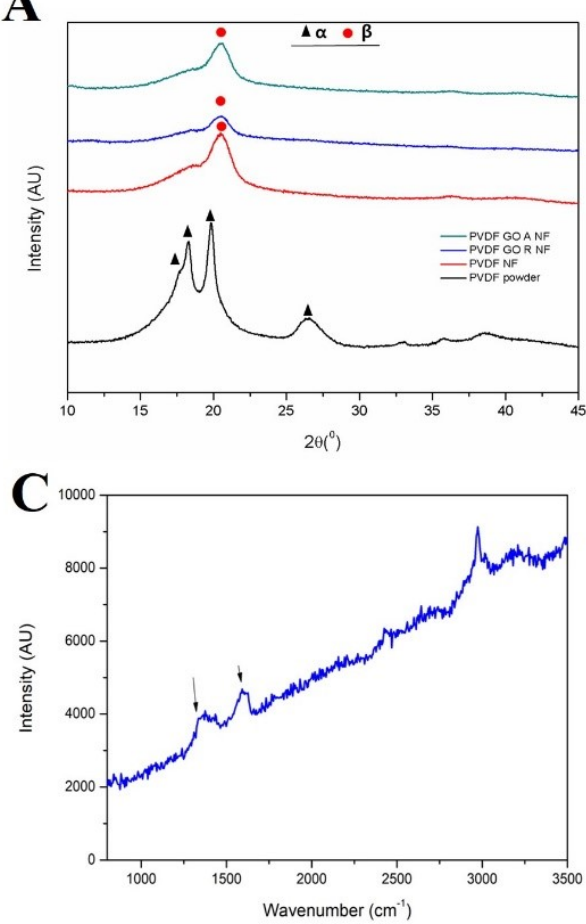

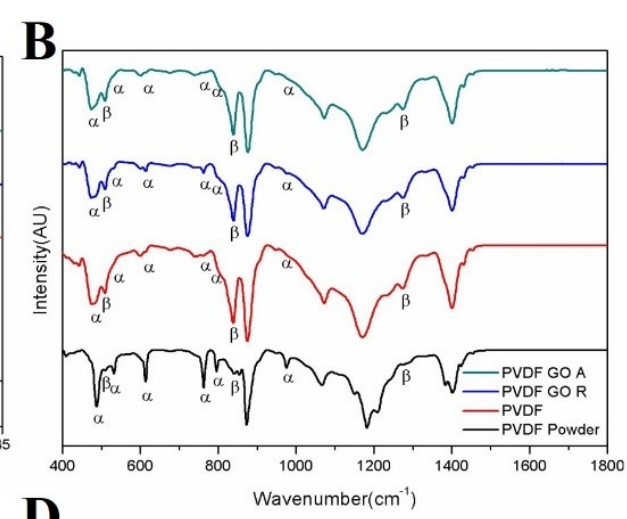

D

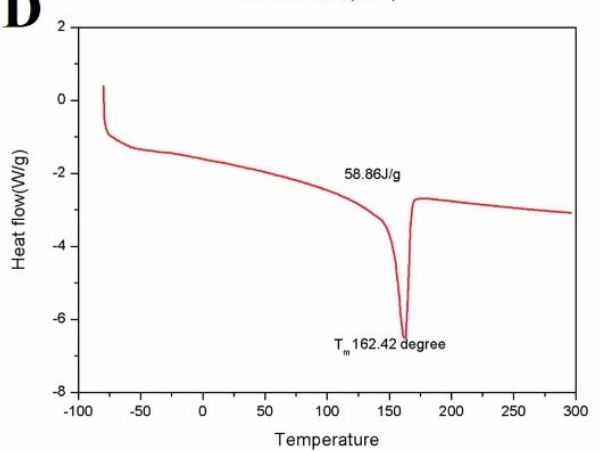

Figure 2. (A) XRD, (B) FTIR showing the $\beta$ phase nucleation in the NF samples, (C) Raman spectra showing the characteristic peak of GO in the PGANF, (D) DSC thermograph of PGANF showing the melting temperature Tm.

The piezoelectric characteristics of the prepared samples were analysed by fabricating the NG as described earlier [43]. The optical image revealing the flexibility is given in Figure S4 of SI. The device is ready for piezoelectric characterisation. A further poling step is not required in the case of electrospun samples, as the electrospinning technique itself produce poling in the samples by the presence of electric field and the mechanical stretching [44,45]. Figure 3A shows the set up for the electrical characterisation of the FENG. The mechanical force is given by the help of a custom-made motor device and the mechanical force was controlled at different frequencies of vibrations. Figure 4 shows the piezoelectric response of the nanofiber samples prepared as part of the study. For that, the NG designs were made as described above and moderately high mechanical force with a frequency of $8 \mathrm{~Hz}$ with a uniform force of $12 \mathrm{~N}$ was applied across the PGANF, PGRNF and PVADF NFs FENGs respectively. The piezoelectric potential generated across the electrode was measured using an oscilloscope (Yokogawa, DL9040). As expected, PGANF exhibited maximum piezoelectric potential $2.1 \mathrm{~V}$ compared to the PGRNF (1.5V) and PVDF NF (0.62V) FENGs. This can be attributed to the difference in the $\beta$ phase nucleation in the different NF samples, which is directly related to the change in the piezoelectricity [46]. The $d_{33}$ value is calculated from output charge and applied mechanical force for the following samples: PVDF R, PVDF GO R, and PVDF GO A as 3.64, 8.75, and $12.25 \mathrm{pC} / \mathrm{N}$, respectively. The results clearly confirm that the piezo potential of the sample increase with increasing polar $\beta$ phase in the nanocomposite.

The output voltage as a function of the mechanical vibration frequency was analysed for the PGANF (Figure 5A). It was found that after application of an external force with a low cyclic frequency of $2 \mathrm{~Hz}$ across the PGANF FENG resulted in a low piezoelectric potential, whereas the high cyclic frequency of $8 \mathrm{HZ}$ induced a maximum piezoelectric potential. In a typical experiment, the PGANF FENG exhibited a piezoelectric voltage of $2.1 \mathrm{~V}$ when applied with a high mechanical frequency of 8 $\mathrm{Hz}$, whereas it exhibited a lower piezoelectric voltage of $0.648 \mathrm{~V}$ for a small mechanical vibration with $2 \mathrm{~Hz}$ frequency (Figure S5 of SI). This is due to the particular orientation of the electric dipoles in the NF due to the stress-induced poling effect [47]. Figure 5B shows that the cyclic loading and unloading on the piezoelectric device with $12 \mathrm{~N}$ force at a constant $8 \mathrm{~Hz}$ frequency generates a piezoelectric 
potential of $2 \mathrm{~V}$ steadily for more than 2000 cycles (Figure 5B insert shows a detailed magnified plot of generated piezoelectric potential during cyclic loading). This result depicts the durability and mechanical stability of a fabricated low-cost piezoelectric device, which could be used for long term biomechanical piezoelectric sensor applications. This remarkable durability confirms the applicability of PGANF FENG.

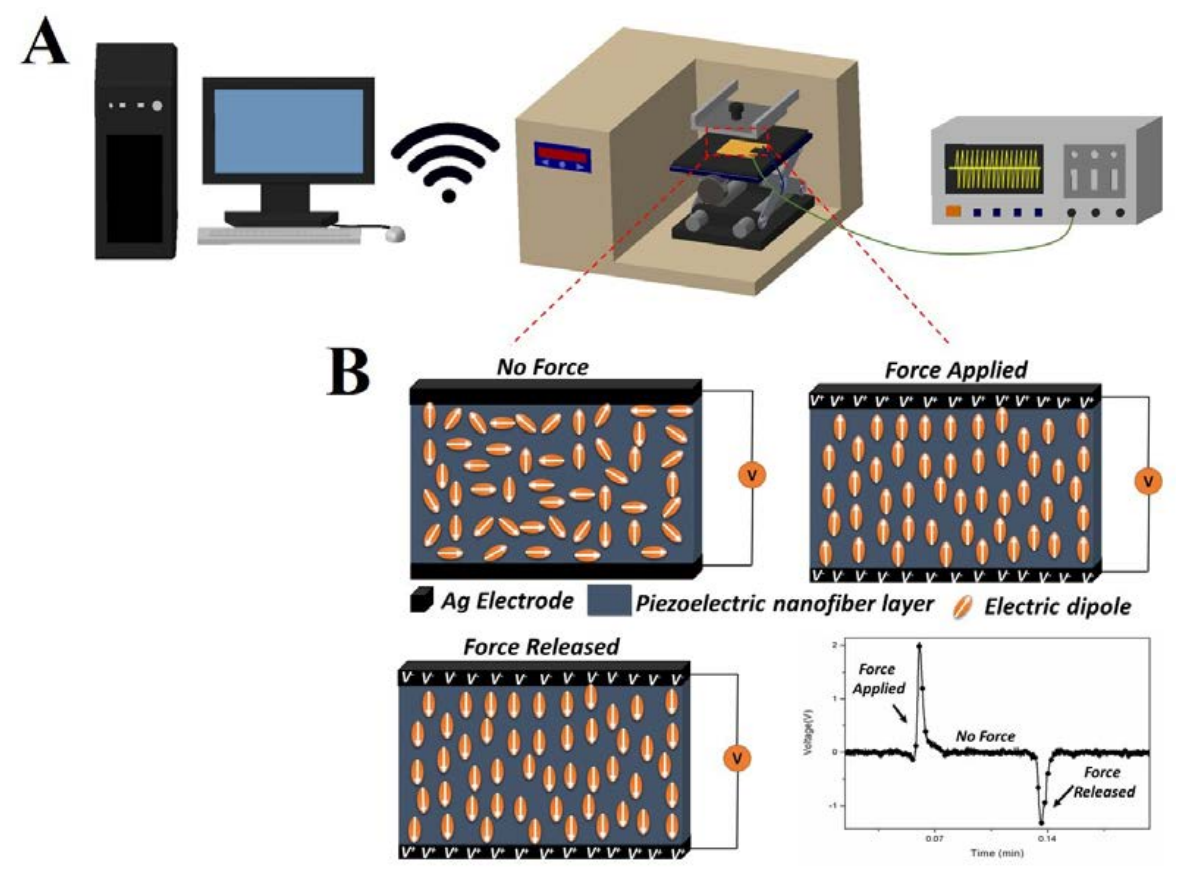

Figure 3. Schematic illustration showing. (A) the experimental set up for piezoelectricity measurement, (B) schematic illustration showing the working mechanism of the PGANF ferroelectric nanogenerator (FENG).
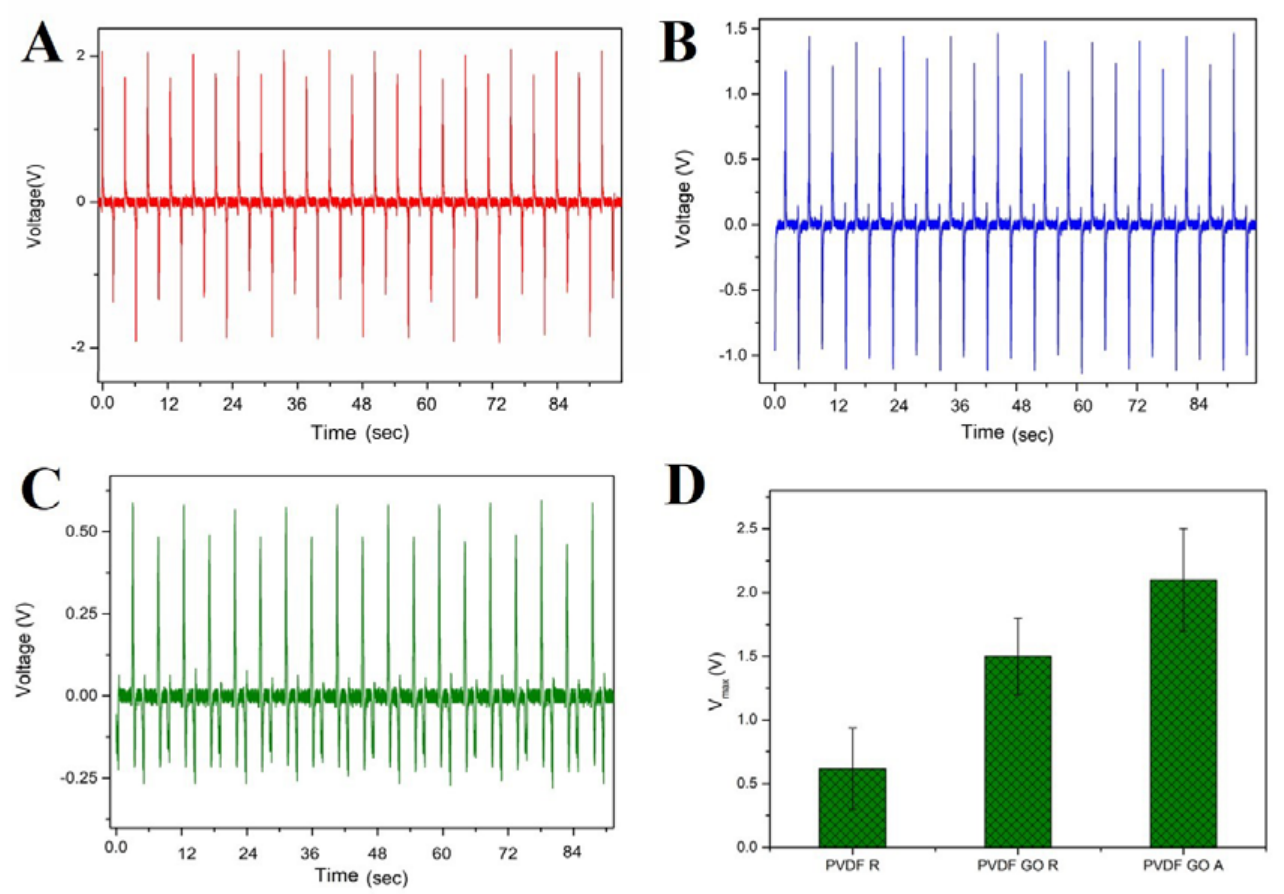

Figure 4. Electrical response of the nanogenerators under a constant mechanical vibration frequency of $8 \mathrm{~Hz}$ and a uniform force of 12 N. (A) PGANF, (B) PGRNF, (C) PNF, (D) Comparison of the maximum output voltage. 

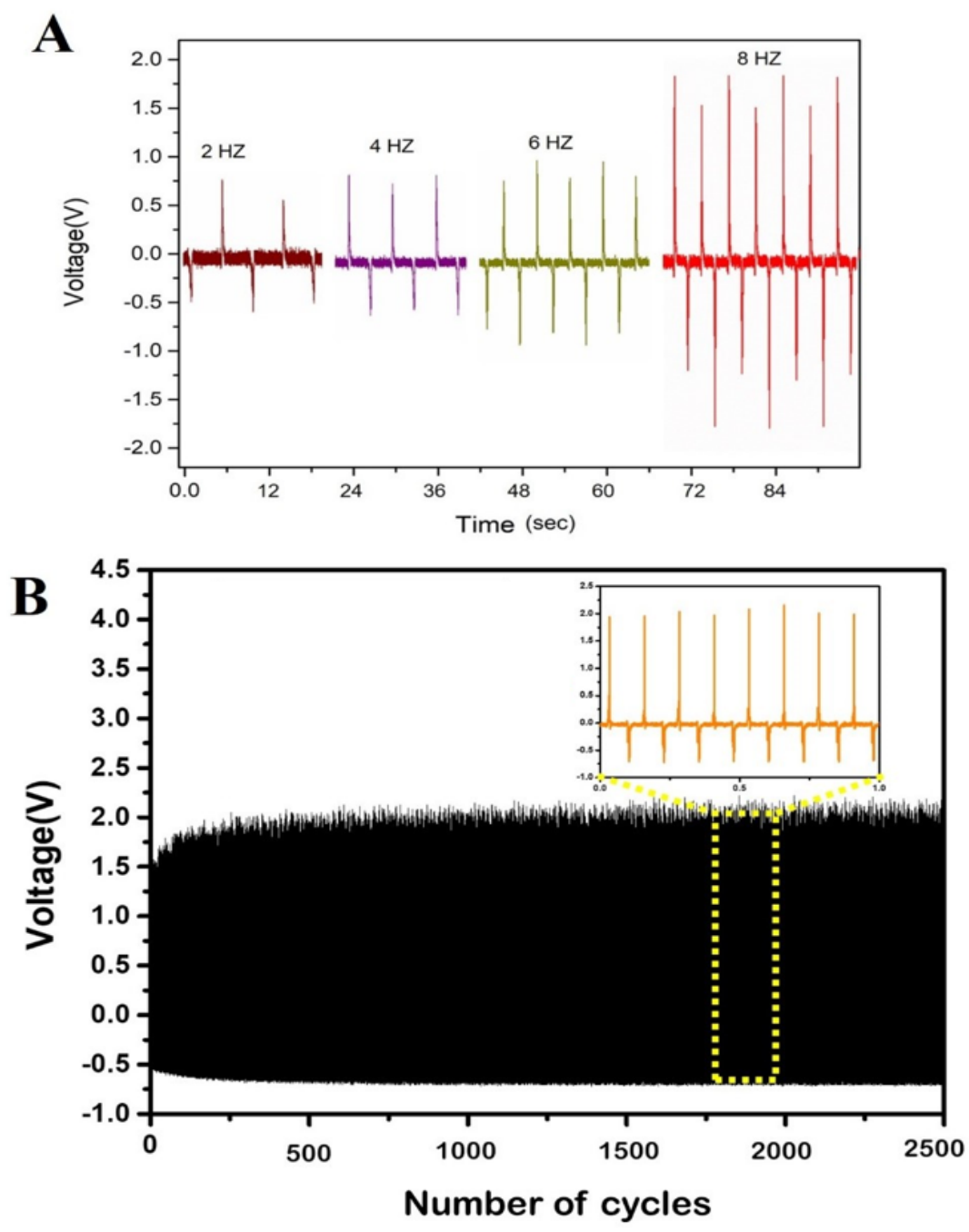

Figure 5. (A) The piezoelectric voltage generated from the PGANF FENG under different mechanical vibration frequency, (B) Mechanical durability test of the PGANF FENG under repeated pushing and no pushing conditions with frequency of $8 \mathrm{~Hz}$ at a uniform force of $12 \mathrm{~N}$.

After confirming the direct piezoelectricity and durability of PGANF FENG, we set out to investigate its efficacy to recover biomechanical energy. Starting from a low frequency biomechanical motion of finger stretching and relaxation to high frequency locomotion, sensing was performed. As per the study for the finger movement, the PGANF FENG device generated a piezoelectric output voltage of $0.08 \mathrm{~V}$, confirming the efficacy of the proposed device for the real-time non-invasive monitoring of the human physiological signals (Figure 6). We also tested the efficacy of the PGANF FENG device to harness the energy from our movements while sitting in a chair called a smart chair. Here, we attached the prototype PGANF FENG device onto the handle of an office chair and the frequent hand pushes evoked a piezoelectric output potential of 1.9 V. Further application of the PGANF FENG device was extended to analyse as a locomotion detector. The output power obtained from the device attached on the sandal exhibited a very high mechanical strength as well as comparable piezoelectric output potential of $6 \mathrm{~V}$. 
A

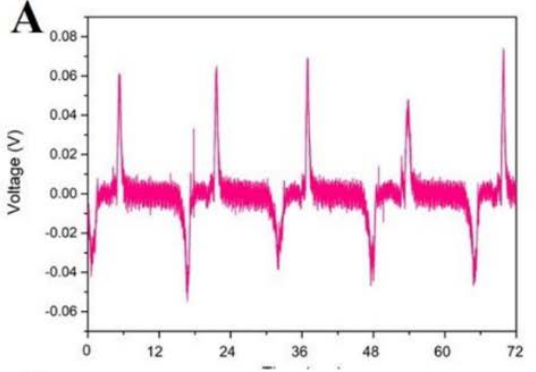

C

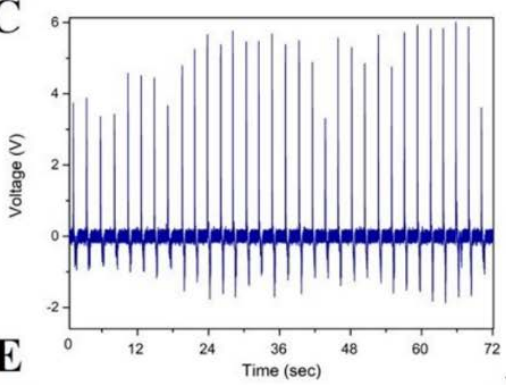

$\mathbf{E}$

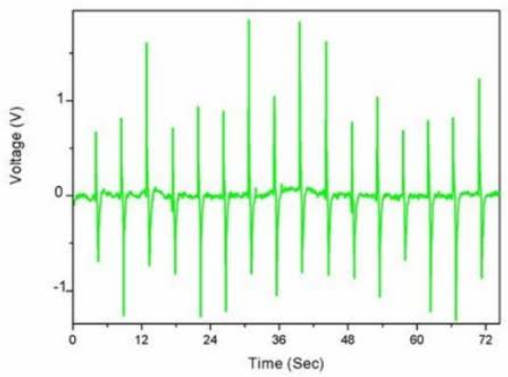

B

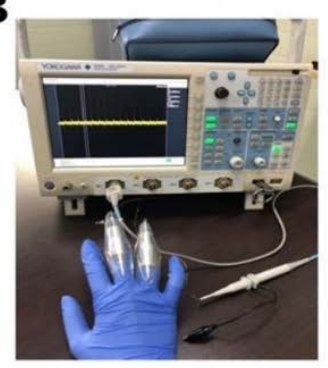

D

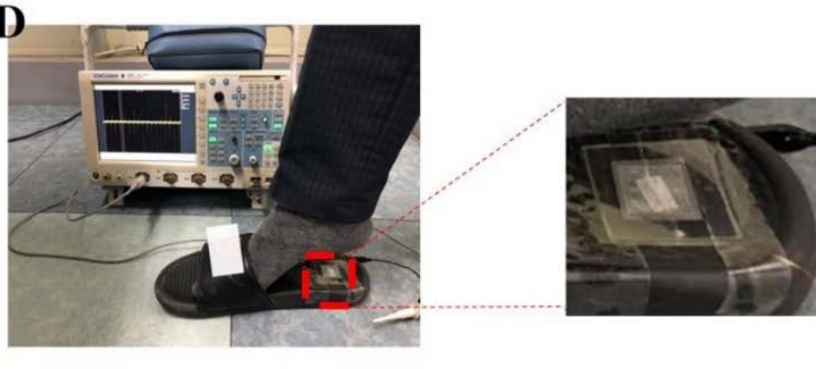

$\mathbf{F}$

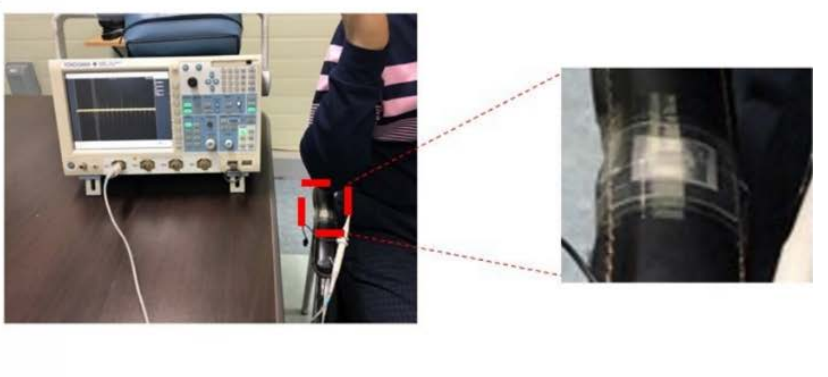

Figure 6. Biomechanical piezoelectric sensors studies: Piezoelectric output voltage and photograph of PGANF FENG as (A,B) Bio E-Skin, (C,D) locomotion detector, (E,F) smart chair.

\section{Conclusions}

In summary, we have successfully prepared PVDF and PVDF GO NFs by the electrospinning method. The effect of nanofiller and the alignment of the nanofibers on the nucleation of electroactive $\beta$ phase also evaluated and found that GO-loaded aligned NF exhibited increased $\beta$ phase compared to the GO-loaded random NFs and pristine PVDF nanofibers due to the active interaction of GO with the PVDF matrix and the uniaxial stretching. The structural characterisation using XRD and FTIR confirms the enhanced nucleation and stabilised polar $\beta$ phase. The PGANF exhibited the highest piezoelectric output voltage of $2.1 \mathrm{~V}$ and it was successfully tested its efficacy for biomechanical piezoelectric sensors as a glove sensor, smart chair or locomotion detector. The enhanced piezoelectric efficiency suggests the possibility of these nanofibers in electronic and biomedical applications.

Supplementary Materials: The following are available online at http://www.mdpi.com/2076-3417/10/10/3493/s1, Figure S1: FESEM image showing the PVDF GO NF, Figure S2: FTIR showing the characteristic peak of GO in the PGRNF and PGANF. Figure S3: Comparison of the relative proportion of $\beta$ phase obtained from both XRD and FTIR. Figure S4: Photograph showing the flexibility of PEGANF FENG's, Figure S5: The piezoelectric voltage generated from the PEGANF FENG under different mechanical vibration frequency.

Author Contributions: A.R.K.S. designed the research and M.K. conducted the experiments. V.K.K. assisted M.K. to fabricate FENG device. A.R.U. guided in the overall electrospinning process. M.K. and A.R.K.S. wrote the manuscript. C.H.P. and C.S.K. conducted the overall analysis and guided the experiments. All authors have read and agreed to the published version of the manuscript.

Funding: This paper was supported by grant from the Basic Science Research Program through National Research Foundation of Korea (NRF) by Ministry of Education, Science and Technology (Project no. 2016R1D1A1B03934124 and 2019R1A2C1003988) and also partially supported by the program for fostering next-generation researchers 
in engineering of National Research Foundation of Korea (NRF) funded by the Ministry of Science, (Project no. 2017H1D8A2030449).

Conflicts of Interest: The authors declare no conflict of interest.

\section{References}

1. Dagdeviren, C.; Li, Z.; Wang, Z.L. Energy Harvesting from the Animal/Human Body for Self-Powered Electronics. Annu. Rev. Biomed. Eng. 2017, 19, 85-108. [CrossRef] [PubMed]

2. Guyomar, D.; Badel, A.; Lefeuvre, E.; Richard, C. Toward energy harvesting using active materials and conversion improvement by nonlinear processing. IEEE T. Ultrason. Ferroelectr. 2005, 52, 584-595.

3. Wang, Z.L. Piezopotential gated nanowire devices:Piezotronics and piezo-phototronics. Nano Today 2010, 5 , 512-514. [CrossRef]

4. Hinchet, R.; Kim, S.W. Wearable and Implantable Mechanical Energy Harvesters for Self-Powered Biomedical Systems. Acs Nano 2015, 9, 7742-7745. [CrossRef] [PubMed]

5. Wu, F.; Yu, P.; Mao, L.Q. Self-powered electrochemical systems as neurochemical sensors: Toward self-triggered in vivo analysis of brain chemistry. Chem. Soc. Rev. 2017, 46, 2692-2704. [CrossRef] [PubMed]

6. Lee, M.; Bae, J.; Lee, J.; Lee, C.S.; Hong, S.; Wang, Z.L. Self-powered environmental sensor system driven by nanogenerators. Energy Environ. Sci. 2011, 4, 3359-3363. [CrossRef]

7. Fan, F.R.; Tang, W.; Wang, Z.L. Flexible Nanogenerators for Energy Harvesting and Self-Powered Electronics. Adv. Mater. 2016, 28, 4283-4305. [CrossRef]

8. Wang, Z.L.; Song, J.H. Piezoelectric nanogenerators based on zinc oxide nanowire arrays. Science 2006, 312, 242-246. [CrossRef]

9. Cha, S.N.; Seo, J.S.; Kim, S.M.; Kim, H.J.; Park, Y.J.; Kim, S.W.; Kim, J.M. Sound-driven piezoelectric nanowire-based nanogenerators. Adv. Mater. 2010, 22, 4726. [CrossRef]

10. Park, K.I.; Xu, S.; Liu, Y.; Hwang, G.T.; Kang, S.J.L.; Wang, Z.L.; Lee, K.J. Piezoelectric $\mathrm{BaTiO}_{3}$ thin film nanogenerator on plastic substrates. Nano Lett. 2010, 10, 4939-4943. [CrossRef]

11. Xu, S.; Hansen, B.J.; Wang, Z.L. Piezoelectric-nanowire-enabled power source for driving wireless microelectronics. Nat. Commun. 2010, 1, 1-5. [CrossRef]

12. Kumari, P.; Rai, R.; Sharma, S.; Shandilya, M.; Tiwari, A. State-of-the-art Of Lead Free Ferroelectrics: A Critical Review. Adv. Mater. Lett. 2015, 6, 453-484. [CrossRef]

13. Hwang, G.T.; Park, H.; Lee, J.H.; Oh, S.; Park, K.I.; Byun, M.; Park, H.; Ahn, G.; Jeong, C.K.; No, K.; et al. Self-powered cardiac pacemaker enabled by flexible single crystalline PMN-PT piezoelectric energy harvester. Adv. Mater. 2014, 26, 4880. [CrossRef] [PubMed]

14. Rakbamrung, P.; Lallart, M.; Guyomar, D.; Muensit, N.; Thanachayanont, C.; Lucat, C.; Guiffard, B.; Petit, L.; Sukwisut, P. Performance comparison of PZT and PMN-PT piezoceramics for vibration energy harvesting using standard or nonlinear approach. Sens. Actuat a-Phys. 2010, 163, 493-500. [CrossRef]

15. Kwon, J.; Seung, W.; Sharma, B.K.; Kim, S.W.; Ahn, J.H. A high performance PZT ribbon-based nanogenerator using graphene transparent electrodes. Energy Environ. Sci. 2012, 5, 8970-8975. [CrossRef]

16. Do, Y.H.; Jung, W.S.; Kang, M.G.; Kang, C.Y.; Yoon, S.J. Preparation on transparent flexible piezoelectric energy harvester based on PZT films by laser lift-off process. Sens. Actuat A-Phys. 2013, 200, 51-55. [CrossRef]

17. Park, K.I.; Lee, M.; Liu, Y.; Moon, S.; Hwang, G.T.; Zhu, G.; Kim, J.E.; Kim, S.O.; Kim, D.K.; Wang, Z.L.; et al. Flexible nanocomposite generator made of $\mathrm{BaTiO}_{3}$ nanoparticles and graphitic carbons. Adv. Mater. 2012, 24, 2999-3004. [CrossRef] [PubMed]

18. Chang, J.Y.; Domnner, M.; Chang, C.; Lin, L.W. Piezoelectric nanofibers for energy scavenging applications. Nano Energy 2012, 1, 356-371. [CrossRef]

19. Panda, P.K. Review: Environmental friendly lead-free piezoelectric materials. J. Mater. Sci 2009, 44, 5049-5062. [CrossRef]

20. Siddiqui, S.; Kim, D.I.; Duy, L.T.; Nguyen, M.T.; Muhammad, S.; Yoon, W.S.; Lee, N.E. High-performance flexible lead-free nanocomposite piezoelectric nanogenerator for biomechanical energy harvesting and storage. Nano Energy 2015, 15, 177-185. [CrossRef] 
21. Tien, N.T.; Hung, T.Q.; Seoul, Y.G.; Kim, D.I.; Lee, N.E. Physically Responsive Field-Effect Transistors with Giant Electromechanical Coupling Induced by Nanocomposite Gate Dielectrics. Acs Nano 2011, 5, 7069-7076. [CrossRef] [PubMed]

22. Jain, A.; Prashanth, K.J.; Sharma, A.K.; Jain, A.; Rashmi, P.N. Dielectric and piezoelectric properties of PVDF/PZT composites: A review. Polym. Eng. Sci. 2015, 55, 1589-1616. [CrossRef]

23. Shin, S.H.; Kim, Y.H.; Lee, M.H.; Jung, J.Y.; Nah, J. Hemispherically aggregated BaTiO3 nanoparticle composite thin film for high-performance flexible piezoelectric nanogenerator. Acs Nano 2014, 8, 2766-2773. [CrossRef]

24. Bhavanasi, V.; Kusuma, D.Y.; Lee, P.S. Polarization Orientation, Piezoelectricity, and Energy Harvesting Performance of Ferroelectric PVDF-TrFE Nanotubes Synthesized by Nanoconfinement. Adv. Energy Mater. 2014, 4, 1400723. [CrossRef]

25. Holmes-Siedle, A.G.; Wilson, P.D.; Verrall, A.P. PVdF: An electronically-active polymer for industry. Mater. Des. 1984, 4, 910-918. [CrossRef]

26. Salimi, A.; Yousefi, A.A. Analysis Method: FTIR studies of $\beta$-phase crystal formation in stretched PVDF films. Polym. Test. 2003, 22, 699-704. [CrossRef]

27. Nunes, J.S.; Sencadas, V.; Wu, A.; Vilarinho, P.M.; Lanceros-Mendez, S. Piezoelectric and optical response of uniaxially stretched (VDF/TrFE) (75/25) copolymer films. Mater. Sci. Forum 2006, 514-516, 915-919.

28. Sencadas, V.; Moreira, V.M.; Lanceros-Mendez, S.; Pouzada, A.S.; Gregorio, R. alpha-to-beta transformation on PVDF films obtained by uniaxial stretch. Mater. Sci. Forum 2006, 514-516, 872-876. [CrossRef]

29. Karan, S.K.; Bera, R.; Paria, S.; Das, A.K.; Maiti, S.; Maitra, A.; Khatua, B.B. An Approach to Design Highly Durable Piezoelectric Nanogenerator Based on Self-Poled PVDF/AlO-rGO Flexible Nanocomposite with High Power Density and Energy Conversion Efficiency. Adv. Energy Mater. 2016, 6, 1601016. [CrossRef]

30. Chang, C.E.; Tran, V.H.; Wang, J.B.; Fuh, Y.K.; Lin, L.W. Direct-write piezoelectric polymeric nanogenerator with high energy conversion efficiency. Nano Lett. 2010, 10, 726-731. [CrossRef]

31. Karan, S.K.; Mandal, D.; Khatua, B.B. Self-powered flexible Fe-doped RGO/PVDF nanocomposite: An excellent material for a piezoelectric energy harvester. Nanoscale 2015, 7, 10655-10666. [CrossRef] [PubMed]

32. Zheng, J.F.; He, A.H.; Li, J.X.; Han, C.C. Polymorphism Control of Poly(vinylidene fluoride) through Electrospinning. Macromol. Rapid Commun. 2007, 28, 2159-2162. [CrossRef]

33. Wang, Y.R.; Zheng, J.M.; Ren, G.Y.; Zhang, P.H.; Xu, C. A flexible piezoelectric force sensor based on PVDF fabrics. Smart Mater. Struct. 2011, 20, 045009. [CrossRef]

34. Farrar, D.; Ren, K.L.; Cheng, D.; Kim, S.; Moon, W.; Wilson, W.L.; West, J.E.; Yu, S.M. Permanent Polarity and Piezoelectricity of Electrospun alpha-Helical Poly(alpha-Amino Acid) Fibers. Adv. Mater. 2011, $23,3954$. [CrossRef]

35. Sasikala, A.R.K.; Unnithan, A.R.; Thomas, R.G.; Ko, S.W.; Jeong, Y.Y.; Park, C.H.; Kim, C.S. Multifaceted Implantable Anticancer Device for Potential Postsurgical Breast Cancer Treatment: A Single Platform for Synergistic Inhibition of Local Regional Breast Cancer Recurrence, Surveillance, and Healthy Breast Reconstruction. Adv. Funct. Mater. 2018, 28, 1704793. [CrossRef]

36. Sasikala, A.R.K.; Unnithan, A.R.; Yun, Y.H.; Park, C.H.; Kim, C.S. An implantable smart magnetic nanofiber device for endoscopic hyperthermia treatment and tumor-triggered controlled drug release. Acta Biomater. 2016, 31, 122-133. [CrossRef]

37. Cho, S.; Lee, J.S.; Jang, J. Poly(vinylidene fluoride)/NH2-Treated Graphene Nanodot/Reduced Graphene Oxide Nanocomposites with Enhanced Dielectric Performance for Ultrahigh Energy Density Capacitor. Acs Appl. Mater. Interface 2015, 7, 9668-9681. [CrossRef]

38. Abbasipour, M.; Khajavi, R.; Yousefi, A.A.; Yazdanshenas, M.E.; Razaghian, F. The Piezoelectric Response of Electrospun Pvdf Nanofibers with Graphene Oxide, Graphene, and Halloysite Nanofillers: A Comparative Study. J. Mater. Sci-Mater. Electron. 2017, 28, 15942-15952. [CrossRef]

39. Bafqi, M.S.S.; Bagherzadeh, R.; Latifi, M. Nanofiber alignment tuning: An engineering design tool in fabricating wearable power harvesting devices. J. Ind. Text. 2017, 47, 535-550. [CrossRef]

40. Zaarour, B.; Zhu, L.; Jin, X.Y. Controlling the surface structure, mechanical properties, crystallinity, and piezoelectric properties of electrospun PVDF nanofibers by maneuvering molecular weight. Soft Mater. 2019, 17, 181-189. [CrossRef]

41. Wu, D.Z.; Huang, S.H.; Xiao, Z.M.; Yu, L.K.; Wang, L.Y.; Sun, D.H.; Lin, L.W. Piezoelectric Properties of PVDF Nanofibers via Non-uniform Field Electrospinning. Int. Conf. Manip. Manu 2014, 285-289. 
42. Xie, G.Q.; Xi, P.X.; Liu, H.Y.; Chen, F.J.; Huang, L.; Shi, Y.J.; Hou, F.P.; Zeng, Z.Z.; Shao, C.W.; Wang, J. A facile chemical method to produce superparamagnetic graphene oxide-Fe3O4 hybrid composite and its application in the removal of dyes from aqueous solution. J. Mater. Chem. 2012, 22, 25485. [CrossRef]

43. Bhavanasi, V.; Kumar, V.; Parida, K.; Wang, J.X.; Lee, P.S. Enhanced Piezoelectric Energy Harvesting Performance of Flexible PVDF-TrFE Bilayer Films with Graphene Oxide. Acs Appl. Mater. Interfaces 2016, 8, 521-529. [CrossRef] [PubMed]

44. Li, L.; Zhang, M.Q.; Rong, M.Z.; Ruan, W.H. Studies on the transformation process of PVDF from alpha to beta phase by stretching. Rsc Adv. 2014, 4, 3938-3943. [CrossRef]

45. El Mohajir, B.E.; Heymans, N. Changes in structural and mechanical behaviour of PVDF with processing and thermomechanical treatments. 1. Change in structure. Polymer 2001, 42, 5661-5667. [CrossRef]

46. Mao, Y.; Zhao, P.; McConohy, G.; Yang, H.; Tong, Y.; Wang, X. Sponge-Like Piezoelectric Polymer Films for Scalable and Integratable Nanogenerators and Self-Powered Electronic Systems. Adv. Energy Mater. 2014, 4, 1301624. [CrossRef]

47. Alluri, N.R.; Saravanakumar, B.; Kim, S.J. Flexible, Hybrid Piezoelectric Film (BaTi(1-x)Zr(x)O3)/PVDF Nanogenerator as a Self-Powered Fluid Velocity Sensor. Acs Appl. Mater. Interface 2015, 7, 9831-9840. [CrossRef] [PubMed]

(C) 2020 by the authors. Licensee MDPI, Basel, Switzerland. This article is an open access article distributed under the terms and conditions of the Creative Commons Attribution (CC BY) license (http://creativecommons.org/licenses/by/4.0/). 